\title{
Especificaciones del servicio de cesación tabáquica
}

Elaborado por:

Ana Mendoza, Belén Cobián, Jesús Gómez, Miguel Cano, Vicente J. Baixauli (coordinador)

Comisión de Servicios Profesionales Farmacéuticos de SEFAC.

\section{Revisado por:}

\section{Javier Plaza, Laura Gandía, Montse Moral, Xavier Boleda}

Grupo de trabajo de Respiratorio de SEFAC.

\section{RESUMEN}

En abril de 2013, la Sociedad Española de Farmacia Familiar y Comunitaria (SEFAC) presentó su propuesta sobre servicios profesionales farmacéuticos (SPF) cuyo fin es cubrir las necesidades relacionadas tanto con la atención de los pacientes que utilizan medicamentos, como con la salud pública. Esta propuesta ofrece un planteamiento sobre la implantación y desarrollo de los SPF con el objetivo de impulsar su prestación por las farmacias comunitarias en los próximos años. De acuerdo con dicha propuesta todos los SPF que constituyen el catálogo de servicios contarán con un documento de especificaciones.

El objeto de este documento de especificaciones, aprobado por la Junta Directiva de SEFAC el 20 de abril de 2014, es definir y caracterizar el servicio de cesación tabáquica con una doble finalidad:

- Ayudar al farmacéutico comunitario y a sus representantes en el ofrecimiento, prestación, difusión, financiación y concertación de este servicio.

- Servir de guía a los farmacéuticos comunitarios que desean implantar un servicio de cesación tabáquica en la farmacia o elaborar un procedimiento normalizado de trabajo para su realización.

Este documento se complementa con el programa CESAR de formación en cesación tabáquica, y el Documento de intervención en Cesación tabáquica en farmacia comunitaria elaborado y avalado por SEFAC y validado por SEFAC, la Sociedad Española de Neumología y Patología Torácica (SEPAR), la Sociedad Española de Especialistas en Tabaquismo (SEDET) y las sociedades médicas de atención primaria (semFYC, SEMG y SEMERGEN).

\section{ABSTRACT \\ Specifications for the smoking cessation service}

In April 2013, the Spanish Society of Family and Community Pharmacies ( SEFAC ) presented its proposal for pharmaceutical professional services (PPS) which aims to cover needs related to both care for patients using drugs, and public health. This proposal offers an approach for implementing and developing PPS in order to boost its provision by community pharmacies in the coming years. According to this proposal, all PPS comprising the service catalogue will have a specifications document.

The purpose of this specifications document, approved by the Board of SEFAC the April 20, 2014, is to define and provide specifics on the smoking cessation service, with a dual purpose:

- To help community pharmacists and their representatives in the offering, delivery, distribution, financing and coordination of this service.

- Serve as a guide to community pharmacists who wish to implement a smoking cessation service at the pharmacy or develop a standard working procedure for implementation of same.

This document is complemented by the CESAR training program on smoking cessation, and the Document on intervention in Smoking Cessation in community pharmacies prepared and endorsed by SEFAC and validated by SEFAC, the Spanish Society of Pneumology and Thoracic Pathology (SEPAR) the Spanish Society of Smoking Specialists (SEDET) and primary care medical associations (SemFYC, SEMG and SEMERGEN).

Financiación: No se ha contado con financiación para la realización de este documento.

Conflicto de intereses: Los autores declaran no existir conflicto de intereses en relación con el contenido del documento. Cite este artículo como: Mendoza A, Cobián B, Gómez J, Cano M, Baixauli VJ. Especificaciones del servicio de cesación tabáquica. Farmacéuticos Comunitarios. 2016 Mar 01; 8(1):32-38. doi:10.5672/FC.2173-9218.(2016/Nol8).001.05 Correspondencia: sefac@sefac.org.

ISSN 1885-8619 @ SEFAC (Sociedad Española de Farmacia Familiar y Comunitaria). Todos los derechos reservados. 


\section{Introducción y justificación}

La Sociedad Española de Farmacia Familiar y Comunitaria (SEFAC) en su propuesta sobre servicios profesionales farmacéuticos (SPF) (1) se compromete a impulsar la implantación y desarrollo de los SPF con el objetivo de facilitar su implantación por las farmacias comunitarias.

El tabaquismo es una enfermedad crónica, adictiva y recidivante reconocida como trastorno mental y del comportamiento por la Organización Mundial de la Salud (OMS) (2-4), siendo además la primera causa evitable de morbilidad y mortalidad en el mundo desarrollado $(5,6)$. Frecuentemente el consumo de tabaco da lugar a una dependencia, comparable a la producida por opiáceos, alcohol o cocaína, pero existen intervenciones clínicas efectivas para contrarrestarla (6). Los profesionales sanitarios deben, en primer lugar, ayudar a sus pacientes a comprender el riesgo que supone el consumo de tabaco para su salud y facilitar que se planteen la necesidad de abandonarlo, pero además deben atender al fumador en el proceso de cesación y ayudarle a superar las posibles recaídas (7).

Las farmacias comunitarias son uno de los marcos adecuados para prestar este servicio. El farmacéutico es el profesional sanitario más accesible y las farmacias españolas son visitadas diariamente por cientos de miles de personas. Son, por lo tanto, un punto importante tanto en campañas de educación sanitaria para la población general, como en servicios de cesación tabáquica para fumadores.

Pero además, se ha demostrado la eficacia de una intervención mínima (8) para concienciar al fumador de que no solo es importante, para su salud y la de los suyos dejar de fumar, sino que además, es posible. El equipo de profesionales con el que cuenta la farmacia tiene que estar preparado para proporcionarla.

Por otra parte, la atención individualizada al fumador decidido a dejar de fumar, generadora de un soporte emocional y farmacológico (en caso necesario), tiene que ser prestada por farmacéuticos capacitados para ello.

\section{Definición y descripción breve}

El servicio de cesación tabáquica es el servicio profesional, realizado en la farmacia comunitaria por un farmacéutico capacitado, encaminado a concienciar, apoyar y acompañar al fumador en el abandono del consumo de tabaco. Se actuará a dos niveles:

Concienciando sobre la importancia de abandonar el consumo de tabaco a aquellos fumadores que no contemplen abandonar la adicción, mediante una intervención mínima.

Ayudando a aquellos fumadores que intentan dejar el consumo, mediante la realización periódica de entrevistas clínicas entre el paciente y el farmacéutico, en las que éste obtiene información sobre las características del paciente en relación con su adicción y proporciona soporte farmacológico y/o consejos cognitivo-conductuales e informa, motiva, monitoriza, interviene y ayuda, según sus necesidades, con el fin de conseguir la cesación tabáquica.

Básicamente el servicio de cesación tabáquica se puede describir en tres procesos, uno general de información sobre el tabaquismo y la oferta del servicio y dos más, individualizados dirigidos específicamente al paciente fumador, atendiendo a su disposición para dejar de fumar: la intervención mínima y/o la atención personalizada continuada.

\section{Objetivos}

1. Objetivos generales

- Eliminar el consumo de tabaco en la población que atiende la farmacia.

- Concienciar, apoyar y acompañar al fumador en el abandono del consumo de tabaco.

\section{Objetivos específicos}

- Proporcionar a la población información sobre los beneficios y la importancia de la cesación tabáquica, así como de los perjuicios para el fumador y los que están a su alrededor.

- Posicionar a la farmacia comunitaria como un espacio de salud donde reflexionar sobre el consumo de tabaco

- Lograr que los fumadores tengan una mayor motivación para dejar de fumar.

- Proveer herramientas eficaces para superar su adicción al tabaco a las personas que quieran dejarlo.

- Lograr el mayor número de intentos y mayores porcentajes de éxito en la cesación tabáquica.

\section{Población. Pacientes diana}

La población diana son los fumadores, ofreciéndoseles distintas intervenciones dependiendo de su disposición a dejar de fumar:

- Fumadores que no deseen dejar de fumar, se realiza una intervención mínima para concienciar y progresar hacia una etapa más avanzada en su decisión de dejar de fumar.

- Fumadores que deseen dejar de fumar, se les ofrece atención personalizada proporcionándoles las herramientas para aumentar la probabilidad de lograrlo.

- Fumadores en proceso de cesación, se les ofrece atención personalizada

\section{Recursos necesarios}

\section{Recursos materiales}

1. Información sobre el consumo de tabaco

La farmacia debe ser un espacio dedicado a reflexionar sobre el consumo de tabaco, por lo que debe difundir mensajes sobre el tabaquismo así como ofertar el servicio de cesación tabáquica.

\section{Zona de Atención Personalizada (ZAP) y zona para terapia grupal.}

Como en toda relación terapéutica, la confianza, la comunicación y la confidencialidad son pilares básicos, por lo que es imprescindible disponer de una zona diferenciada (ZAP) y separada físicamente de la zona de dispensación, que permita la atención al fumador y garantice su intimidad y la confidencialidad de la consulta, sea de forma presencial o a través de otras formas de comunicación (telefónica, correo-electrónico, etc.). 
La ZAP será un espacio de comunicación entre el paciente y el farmacéutico, donde ya sea de forma presencial, telefónica o con otros dispositivos de comunicación, puedan hablar y/o sentarse juntos sin ser escuchados por cualquier otra persona, incluyendo el personal que trabaja en la farmacia.

Para evaluar la conformidad de la ZAP es recomendable seguir los criterios utilizados por Aguiló (9): visibilidad desde la zona de dispensación, accesibilidad, diferenciación del mostrador y otros elementos de la zona de dispensación, privacidad para la comunicación farmacéutico-paciente y un equipamiento mínimo, número de ZAP y zona de espera para los pacientes que deben ser atendidos.

La ZAP contará con el equipamiento necesario para la realización del servicio.

En el caso de realizar terapia grupal para cesación tabáquica también será necesario disponer de un espacio cerrado que permita reunir sentados a todos los pacientes que la requieran y en el que se mantenga la confidencialidad.

\section{Sistema de registro de la documentación.}

Es necesario disponer de un sistema de registro seguro de toda la información utilizada como son los registros, formularios e informes que se manejan en el servicio. Se recomienda disponer de ellos en formato digital para su mejor cumplimentación, archivo y recuperación. También puede ser preciso que el farmacéutico pueda acceder a la información clínica necesaria del paciente tanto a través de los sistemas de informatización sanitaria externos a la farmacia como a la información procedente del programa informático de gestión de la farmacia. De igual forma, puede ser necesario comunicarse con otros profesionales sanitarios e incluso con el paciente.

\section{Fuentes de información sobre medicamentos y deshabituación tabáquica}

Es conveniente disponer de acceso a fuentes de información sobre medicamentos y consulta básica como el BotPlus ${ }^{\circledast}$, etc., así como de guías prácticas de deshabituación tabáquica, programa CESAR de formación en cesación tabáquica, consenso de derivación del fumador, etc.

\section{Equipamiento}

- Báscula.

- Tensiómetro con manguitos de diferentes tamaños.

- Cooxímetro y/o espirómetro (convenientes pero no imprescindibles).

- Cuestionarios específicos (Fagerström, Richmon, etc.).

- Material fungible.

- Cartelería, folletos, etc.

- Teléfono, fax y acceso a internet.

\section{Recursos humanos}

\section{Organización}

La implicación de todo el equipo de la farmacia en el servicio de cesación tabáquica es imprescindible. Hay que establecer claramente las responsabilidades de cada miembro del equipo. A este respecto consideraremos:

Responsable del servicio: Con el fin de organizar mejor este servicio es conveniente que exista un farmacéutico responsable del servicio (10). Cuando en la farmacia sólo ejerza un farmacéutico éste será el responsable del servicio, mientras que en las que trabajen más de uno, se debería nombrar a un responsable del servicio. Su elección se debería realizar en función de sus habilidades de comunicación, su actitud frente al servicio y su experiencia asistencial. El responsable del servicio, junto con el farmacéutico titular, debería establecer los indicadores del servicio, en especial la satisfacción de los pacientes, con el fin de evaluarlos periódicamente.

Continuidad del servicio: Para garantizar la continuidad de este servicio en la farmacia a lo largo del tiempo, es aconsejable que exista más de un farmacéutico capacitado para poder prestarlo, así se evita su suspensión ante la ausencia del farmacéutico capacitado (periodos de vacaciones, bajas por enfermedad...).

\section{Capacitación}

Se trata de un servicio profesional donde la preparación los profesionales resulta imprescindible, por ello todos los farmacéuticos que presten este servicio deberán acreditar que poseen las competencias necesarias para su prestación, por ello deberán estar motivados y capacitados específicamente para prestar este servicio. También deben poseer su correspondiente seguro de responsabilidad civil.

De igual forma es necesario que el resto del personal de la farmacia esté cualificado para proporcionar la intervención mínima, la oferta del servicio y cuente con las instrucciones de derivación correspondientes.

\section{Tiempo}

Este servicio requiere el seguimiento del fumador desde que se inicia el servicio, por lo que es necesaria la concertación de citas periódicas en la farmacia en las que se entrevistan fumador y paciente y se analiza la evolución en la cesación tabáquica, todo ello supone un consumo de tiempo que tiene que proveerse.

\section{Derivación}

En el transcurso del servicio de deshabituación tabáquica, el farmacéutico puede detectar situaciones que excedan los objetivos de este servicio y precisen de una intervención de otro profesional. En estos casos, el farmacéutico indicará al paciente la conveniencia de derivarle a otro servicio, en el que se aborde la situación detectada.

La derivación quedará reflejada en el informe del servicio, así como en el procedimiento normalizado de trabajo (PNT) del servicio, donde se especificarán las situaciones y circunstancias que la determinen. La derivación puede ser de dos tipos: interna y externa.

\section{Derivación interna}

La situación detectada puede tratarse mediante otro servicio disponible en la misma farmacia comunitaria, sea por el mismo profesional u otro del establecimiento. Se registrará el problema, el servicio interno al que se deriva y la justificación de la misma.

\section{Derivación externa}

La situación detectada no puede tratarse en la propia farmacia, por no prestar dicho servicio, exceder las competencias del personal farmacéutico de dicha farmacia, o requerir de otro nivel asistencial, por lo que es preciso 
derivar a otro profesional sanitario. Igualmente se realizará un parte de derivación, previo consentimiento del paciente, adjuntando toda la información necesaria para el otro profesional. El caso más habitual de derivación externa en este servicio es la derivación a:

- Médico de cabecera: es posible que sea necesaria la intervención del médico de cabecera si se considera la utilización de medicamentos sujetos a prescripción médica o si se ve afectado el tratamiento farmacológico del paciente.

- Unidad de tabaquismo: En determinados fumadores, de acuerdo con los criterios de derivación consensuados, puede ser necesario realizar su derivación a una unidad de atención especializada.

\section{Protocolización}

El servicio de cesación tabáquica deberá estar protocolizado y el procedimiento normalizado de trabajo (PNT) deberá ser seguido y conocido por todo el equipo de la farmacia.

El PNT es una herramienta para el profesional, por lo que debe adaptarse a la situación concreta y específica de los profesionales que ejercen en dicha farmacia. Por tanto, sería conveniente que los farmacéuticos de cada farmacia desarrollasen su propio PNT o bien lo adaptaran a su situación particular. En este sentido, SEFAC pondrá a disposición de todos aquellos farmacéuticos que lo soliciten, un PNT de este servicio y establecerá los elementos mínimos que debe reunir cualquier PNT utilizado para el servicio de cesación tabáquica.

\section{Documentación y registros}

La forma de manejar y registrar la información recogida y utilizada en este servicio debe permitir que ésta se introduzca de homogénea, se pueda guardar, consultar, imprimir, interrelacionar y permitir su trazabilidad en relación con los usuarios que acceden a ella.

\section{Registros}

El registro de los datos obtenidos durante el sevicio evidencia su realización y permite demostrar el impacto de la actuación farmacéutica en el paciente y en general en la atención sanitaria prestada a la población. El registro podrá realizarse en soporte papel o preferiblemente en soporte informático y siempre cumplimiendo la normativa en materia de protección de datos (11).

Los registros necesarios para el servicio de cesación tabáquica son los siguientes:

\section{Registros tras la intervención mínima}

No/código de la intervención, grado de motivación, receptividad a la intervención, tiempo que ha de transcurrir para volver a intentarlo.

\section{Registros de atención continuada}

- Entrevista inicial: Información personal del fumador, información sanitaria, valoración de la adicción y recursos personales para afrontarla, peso, presión arterial y cooximetría y espirometría si procede.
- Valoración y plan de actuación: Resultado de la evaluación del caso y plan de actuación acordado

- Primera visita: Registro del día D, Estrategia de tratamiento pactada, Medios para afrontar el síndrome de abstinencia entregados

- Visitas de seguimiento: Registro de peso, presión arterial y cooximetría y espirometría si procede; control de sindrome de abstinencia, cumplimiento del tratamiento, PRM y RNM detectados e intervención para resolverlos.

- Visita final: abandono o exfumador Documentación del servicio:

\section{Documentos de Información general}

Folletos informativos sobre los beneficios de dejar de fumar. Estarán al alcance de todas las personas que entren en la farmacia o incluso de aquellas que pasen por delante de la misma.

\section{Documentación para todo el personal de la farmacia en la intervención mínima}

- Argumentario en la intervención mínima

- Creencias erróneas y barreras que plantean las personas fumadoras

- Diagrama de intervención en cesación tabáquica

\section{Consentimiento informado}

Para la prestación del servicio de cesación tabáquica es imprescindible el consentimiento del paciente. Este consentimiento incluye:

- El tratamiento de sus datos personales de acuerdo con la LOPD.

- El conocimiento sobre el objeto y las características del servicio.

Toda cesión de información sanitaria sobre el paciente a otros profesionales sanitarios requerirá el conocimiento y la autorización del paciente.

\section{Información al fumador en atención personalizada continuada}

- Recomendaciones para el síndrome de abstinencia, ganas de fumar, prevención de recaidas, consejos dietéticos y alimentarios, consejos sobre actividad física, etc.

- Lista de motivos para dejar de fumar.

- Autoregistro de consumo.

\section{Documentación especifica del servicio} para los farmacéuticos

- Pruebas de valoración (Etapa del proceso de cambio según Prochaska-diclemente, Test de Fagerstrom, Richmond, Exploración de la importancia y la confianza, Grado de motivación...).

- Registros clínicos (de intervenciones mínimas, entrevista inicial, primera visita, sucesivas y final, etc.).

- Estrategias para resolución de problemas.

- Modelo de carta de derivación al médico.

\section{Calidad, certificación y mejora continua}

SEFAC participará en todas aquellas acciones e iniciativas que impulsen y aseguren sistemas de gestión y aseguramiento de la calidad de este servicio. 
En este sentido, teniendo en cuenta que la certificación es la acción llevada a cabo por una entidad independiente de las partes interesadas mediante la que se manifiesta que una organización, producto, proceso o servicio, cumple los requisitos definidos en unas normas o especificaciones técnicas (12), SEFAC colaborará en la certificación del servicio en aquellas farmacias que lo demanden.

Hay que distinguir entre 3 tipos de certificaciones:

\section{Certificación de profesionales}

Los farmacéuticos comunitarios que presten el servicio de cesación tabáquica deberán acreditar que cuentan con las competencias necesarias para llevarlo a cabo mediante la certificación de la capacitación específica de este servicio.

\section{Certificación de instalaciones y equipamiento}

Las farmacias comunitarias que presten el servicio cesación tabáquica deberán acreditar que disponen de los recursos materiales necesarios para su prestación: utillaje, instalaciones y equipamiento.

\section{Certificación y validación de Procedimientos Normalizados de Trabajo (PNT)}

Los PNT utilizados en la realización del servicio de cesación tabáquica deben haber sido validados para su utilización en la práctica clínica.

Por último, SEFAC ofrecerá a los farmacéuticos interesados participar en un programa de supervisión externa de la calidad con el fin de poder compararse con otras farmacias y poder mejorar este servicio.

\section{Resultados obtenidos}

Los resultados derivados del servicio de cesación tabáquica deben suponer una mejora tanto clínica como económica, que justifiquen su viabilidad.

Desde el punto de vista clínico, el perjuicio para la salud que causa el tabaco está perfectamente establecido Desde el punto de vista económico, las consecuencias clínicas para la salud del paciente derivadas del tabaquismo en recursos sanitarios y sociales (hospitalizaciones, visitas al médico, pruebas analíticas, etc), son ampliamente conocidas. Estas consecuencias son:

- Causa principal de cáncer y de muerte por cáncer.

- Causa cáncer de pulmón, de esófago, laringe, boca, garganta, riñón, vejiga, páncreas, estómago y de cérvix, así como también leucemia mieloide aguda $(13,14)$.

- Causa enfermedades del corazón, apoplejía, aneurisma de la aorta, enfermedad pulmonar obstructiva crónica, asma, fracturas de cadera y cataratas.

- Quienes fuman presentan un riesgo mayor de padecer neumonía y otras infecciones de las vías respiratorias $(13,14)$.

Tabla 1 Eficacia de la cesación tabáquica en función del tipo de intervención

\begin{tabular}{|l|c|c|c|}
\hline & $\begin{array}{c}\text { Sin } \\
\text { tratamiento }\end{array}$ & $\begin{array}{c}\text { Consejo } \\
\text { breve }\end{array}$ & $\begin{array}{c}\text { Tratamiento } \\
\text { conductual }\end{array}$ \\
\hline Sin fármacos & $5 \%$ & $10 \%$ & $15 \%$ \\
\hline Con fármacos & $10 \%$ & $20 \%$ & $30 \%$ \\
\hline
\end{tabular}

- Las mujeres embarazadas que fuman corren un riesgo mayor de que sus bebés nazcan prematuramente o con peso anormalmente bajo.

- La mujer que fuma durante el embarazo o después de él aumenta el riesgo de que su bebé muera por síndrome de muerte infantil súbita $(13,14)$.

- Los hombres que fuman tienen mayor riesgo de presentar disfunción eréctil (15).

- Los estudios revelan que los fumadores que dejan el tabaco alrededor de los 30 años de edad reducen su probabilidad de morir prematuramente por enfermedades relacionadas con fumar en más de 90\% $(16,17)$.

- Las personas que dejan de fumar alrededor de los 50 años de edad reducen su riesgo de muerte prematura en más de 50\% (17).

- Aun las personas que dejan el tabaco alrededor de los 60 años de edad o más viven más tiempo que quienes siguen fumando (17).

- Inhalar humo de tabaco en el ambiente causa cáncer en personas que no fuman $(13,15)$.

Los resultados obtenidos a través de este servicio tienen una triple dimensión:

\section{Para el paciente}

Supone dejar de fumar, y los beneficios asociados a dejar de fumar. Tanto para el fumador como para sus allegados estos beneficios son indiscutibles. Cuando un fumador realiza un intento de abandono del tabaco sin acompañarlo del tratamiento adecuado, las posibilidades de éxito se cifran entre un 5 a $8 \%$. Sin embargo, este porcentaje se quintuplica cuando el intento se acompaña de un tratamiento adecuado y el apoyo de un profesional sanitario. Los estudios científicos nos informan que los índices de éxito, aun aplicando tratamientos adecuados, se sitúan entre un 15 y un 40\%, lo que nos da una idea de la magnitud del problema. Sin embargo, actualmente no existen datos a nivel de Farmacia en España, si a nivel internacional. Los pocos resultados de los que se dispone de forma preliminar (a 6 meses) son los obtenidos a través de los registros del proyecto CESAR (2) promovido por SEFAC y que muestran un éxito del 71\% (de 132 casos finalizados, 94 dejaron de fumar a los 6 meses, 24 abandonaron y 14 fracasaron pues volvieron a fumar antes de los 6 meses).

La eficacia de los diversos tipos de intervención que pueden realizarse en la farmacia comunitaria es muy variable, aunque no por ello deben dejar de intentarse. En general la eficacia de la cesación en función del tipo de intervención en la cesación tabáquica (18) se muestra en la tabla 1.

\section{Para el farmacéutico}

Supone una oportunidad de ampliar su rol sanitario e incrementa su satisfacción profesional le posibilita una oportunidad de mejora sus habilidades clínicas y de educación para la salud y facilita una mejor relación con otros profesionales sanitarios.

\section{Para el Sistema Sanitario}

Le aporta ahorro económico, al disminuir la morbimortalidad debida al tabaquismo y su coste. 
El importe total de los gastos atribuibles al tabaquismo en España para el año 2008 fue de 14.710 millones de euros y los ingresos por impuestos procedentes del tabaco ese mismo año alcanzaron 9.266 millones de euros, por lo que el tabaquismo costó a los españoles 5.444 millones de euros al año", según la Sociedad Española de Neumología y Cirugía Torácica (SEPAR) (19).

Un estudio de la Universidad de California en San Francisco calcula que entre 1989 y 2008 ha gastado 2.400 millones de dólares (unos 1.780 millones de euros). Pero el trabajo también ha calculado los ahorros en atención sanitaria, lo que representan 134.000 millones (casi 100.000 millones de euros). Es decir, por cada dólar o euro gastado se han ahorrado 55.

\section{Retribución y en su caso financiación}

La implantación, gestión y desarrollo de un servicio de cesación tabáquica requiere de una inversión económica para intentar lograr la consecución de su óptima puesta en marcha y correcto funcionamiento. Esta es la razón del porqué, para establecer la retribución de este servicio, se deben cubrir los costes que ocasionan su realización, y una vez determinados, añadir el beneficio que se pretende obtener.

Los distintos costes requeridos para su prestación son:

\section{Costes fijos (no varian con el número de pacientes atendidos)}

Incluyen los costes derivados de la amortización del aparataje requerido para la realización del servicio: báscula, cooxímetro, espirómetro y tensiómetro (con los tres diferentes manguitos disponibles, según perímetro del brazo del paciente) y en su respectiva proporción de: las inversiones realizadas para poder prestar este servicio (instalaciones, equipamiento y su mantenimiento), de los costes proporcionales de los gastos generales de la farmacia (luz, limpieza, acceso a internet, software, seguros, aplicación LOPD, etc.) y de la capacitación de cesación tabáquica del personal.

\section{Costes variables o gastos de funcionamiento del servicio}

Incluyen los costes del material fungible (papel, tinta,...), cartelería, folletos, hojas para registros, recambios de boquillas para el cooxímetro, o el espirómetro si se utilizan, archivo y la documentación necesaria.

\section{Coste del profesional que el realiza el servicio $y$ depende del tiempo empleado}

Partiendo de la base de que este servicio siempre será prestado por un farmacéutico. La atención continuada de un paciente durante un año se ha cuantificado en 120 minutos, de los cuales la entrevista inicial supone alrededor de 40 minutos y el resto repartidas en visitas sucesivas de 5 minutos, ( 3 durante el primer mes, 2 durante los dos meses siguientes y una al mes hasta completar el año). A este tiempo invertido con el propio paciente, habrá que sumarle el que necesita el farmacéutico, sin la necesaria presencia del paciente, para la realización del volcado de datos, la elaboración de la fase de estudio de la información obtenida y las sucesivas propuestas de intervención tras cada entrevista necesaria. El precio final de este gasto resultará de la relación entre el tiempo medio invertido y el coste económico por hora del farmacéutico calculado en base al convenio de referencia del farmacéutico en cada provincia.

El coste económico relacionado con el tiempo empleado por el farmacéutico, se ha calculado en base al coste por hora de un farmacéutico, que puede variar según los convenios actualmente vigentes a los que está sujeta la farmacia comunitaria, entre 16,97-22,52 euros/hora. De acuerdo con este coste y el tiempo invertido anualmente en el servicio se puede estimar un coste mínimo por farmacéutico de unos 50,91-67,56 euros anuales a lo que habría que añadir la amortización del material y el coste del material desechable.

\section{El beneficio de la farmacia}

La prestación de un servicio, aunque pueda ser obligatorio y éste sea básico, no quiere decir que sea gratuito. La prestación de los SPF implica que estos estén retribuidos y su retribución puede ser diferente en función de sus objetivos, procedimientos, recursos y resultados. Así pues, la financiación de un SPF podría darse por los pacientes/usuarios a los que se les presta, por los proveedores sanitarios (Administración, mutuas, etc.) o mediante sistemas mixtos a modo de copagos. En el caso de que el servicio sea ofrecido a los pacientes particulares y esté financiado o remunerado por ellos, el beneficio final dependerá de la demanda y de la política de precios pactada en función también de los costes o la inversión realizada por cada farmacia para su prestación.

\section{Financiación}

Este servicio no está actualmente financiado en España por el SNS u otras entidades aseguradoras sanitarias. Las intervenciones sanitarias para ayudar a dejar de fumar son coste-efectivas, si las comparamos con otras actividades preventivas $(20,21)$. La cesación del hábito tabáquico en aquellos pacientes incluidos en el servicio, podría suponer un considerable ahorro en costes económicos tanto en recursos sanitarios como sociales (hospitalizaciones, visitas al médico, pruebas analíticas, etc.), ayudando a resolver un verdadero problema de salud pública. Esta relación coste-efectividad del servicio podría, justificar su posible financiación.

\section{Referencias bibliográficas}

1. Baixauli Fernández VJ, Satué de Velasco E, Gil García MI, Roig Sena JC, Villasuso Cores B, Sáenz de Buruaga Pérez de Atxa S. Propuesta de la Sociedad Española de Farmacia Comunitaria (SEFAC) sobre servicios profesionales farmacéuticos en farmacia comunitaria. Farmacéuticos Comunitarios 2013;5(3):119-126.

2. Programa CESAR [Internet]. Capacitación para implantar servicios de cesación tabáquica en la farmacia comunitaria. [Acceso 16/5/2015]. Disponible en http://www.sefac.org/ cesar/

3. Documento de intervención en cesación tabáquica en la farmacia comunitaria. [Internet]. Elaborado y avalado por SEFAC y validado por SEPAR, SEMFYC, SEMERGEN, SEMG Y SEDET. [Acceso 16/5/2015]. Disponible en: http://www. sefac.org 
4. Observatorio para la prevención del tabaquismo. Documento técnico de consenso sobre la atención sanitaria del tabaquismo en España. Madrid: Comité Nacional de Prevención del Tabaquismo (CNPT); 2008.

5. Van Schayck OC, Pinnock H, Ostrem A, Litt J, Tomlins R, Williams S, et al. IPCRG consensus statement: Tackling the smoking epidemia. Practical guidance for primary care. Prim Care Resp J. 2008; 17(3):185-93. doi:10.3132/pcrj.2008.00060

6. The 2008 PHS Guideline Update Panel. Treating Tobacco Use and Dependence: 2008 Update. U.S. Public Health Service Clinical Practice Guideline Executive Summary. Respiratory Care. 2008; 53(9):1217-22.

7. Sociedad Española de Farmacia Comunitaria [Internet]. Programa CESAR. Capacitación para implantar servicios de cesación tabáquica en la farmacia comunitaria. [Acceso 24/9/2015]. Disponible en: http://www.abordajedeltabaquismo.com

8. Sociedad Española de Especialistas en Tabaquismo (SEDET) [Internet]. Intervención interdisciplinar en tabaquismo. Documento de consenso 2010. [Acceso 24/9/2015]. Disponible en: www.sedet.es/webcms/usuario/documentos/2010

9. Aguiló M. Estructura física de la farmacia. En: Faus MJ, Martínez F, editores. Diploma de Atención Farmacéutica. Valencia: General Asde; 1999. Pags. 71-85.

10. Gastelurrutia MA. Castrillon CC. Servicio de Seguimiento Farmacoterapéutico. Curso de implantación y gestión de servicios profesionales en farmacia comunitaria. Madrid: SEFAC; 2011.

11. Cortes Españolas. Ley 15/1999 de 13 de diciembre que regula la protección de datos de carácter personal. Y legislación que la desarrolla. Boletín Oficial del Estado, no 298, (14-121999).

12. Asociación Española de Normalización [Internet]. Certificación. Definición. [Acceso 10-10-2015]. Disponible en: http:// www.aenor.es/aenor/certificacion/procesos/proceso_certificacion_aenor.asp

13. US Department of Health and Human Services [Internet]. How Tobacco Smoke Causes Disease: The Biology and Beha- vioral Basis for Smoking-Attributable Disease. A Report of the Surgeon General. 2010. [Acceso 24/9/2015]. Disponible en: http://www.ncbi.nlm.nih.gov/books/NBK53017/

14. US Department of Health and Human Services [Internet]. The Health Consequences of Smoking. A Report of the Surgeon General. 2004. [Acceso 24/9/2015]. Disponible en: http:// www.cdc.gov/tobacco/data_statistics/sgr/2004/pdfs/whatitmeanstoyou.pdf

15. Austoni E, Mirone V, Parazzini F, Fasolo CB, Turchi P, Pescatori $\mathrm{ES}$, et al. Smoking as a risk factor for erectile dysfunction: data from the Andrology Prevention Weeks 2001-2002. A study of the Italian Society of Andrology (SIA). European Urology 2005;48(5):810-18. doi:10.1016/j.eururo.2005.03.005

16. International Agency for Research on Cancer [Internet]. Tobacco Smoke and Involuntary Smoking. Monographs on the Evaluation of Carcinogenic Risks to Humans.Vol.83, 2004. [Acceso 26/10/2015] Disponible en: http://monographs.iarc. fr/ENG/Monographs/vol83/

17. US Environmental Protection Agency. Respiratory Health Effects of Passive Smoking: lung cancer and other disorders. Washington DC: ORD-OHEA; 1992.

18. Hughes JR. New treatments for smoking cessation. CA Cancer J Clin 2000; 50(3):143-51. doi:10.3322/canjclin.50.3.143

19. Sociedad Española de Neumología y Cirugía Torácica (SEPAR) [Internet]. El tabaquismo cuesta al Estado 5.000 millones al año 29/3/2010 [Acceso 30/10/2015]. Disponible en: http://sociedad.elpais.com/sociedad/2010/03/29/actualidad/1269813609_850215.html

20. Agencia de Evaluación de Tecnologías Sanitarias (AETS) Instituto de Salud Carlos III. Ministerio de Sanidad y Consumo. "Evaluación de la eficacia, efectividad y coste-efectividad de los distintos abordajes terapéuticos para dejar de fumar". Madrid: AETS. Instituto de Salud Carlos III. Septiembre 2003.

21. Documento técnico de consenso sobre la Atención Sanitaria del tabaquismo en España. Observatorio para la prevención del tabaquismo. CNPT. Ministerio de Sanidad y Consumo. 2008. 